Elena Morandi

Università di Bologna

\title{
L'INTERPRETE IN AMBITO GIURIDICO E LA LINGUA CINESE
}

\section{Legal interpreting and the Chinese language}

\begin{abstract}
The interpreter/mediator in the legal field plays a fundamental but very complex role, particularly between very distant languages and cultures, such as the Chinese. Different legal systems presuppose different concepts and roles, and the terminology used does not always correspond to the target language.

Adequate training is therefore necessary for this type of interpreter, since knowledge of the Chinese language and/or interpreting techniques alone is not sufficient when cultural and human factors come into play as in this context.

Keywords: Chinese, Chinese language, Chinese culture, interpreter, legal interpreter

\section{Riassunto}

L'interprete/mediatore in ambito giuridico ricopre un ruolo fondamentale ma molto complesso, in particolare quando si tratta di lingue e culture molto distanti, quale quella cinese. Differenti sistemi giuridici presuppongono concetti e ruoli diversi, e la terminologia impiegata non sempre trova corrispondenza nella lingua di arrivo.

È necessaria quindi un'adeguata formazione per questa tipologia di interpreti, poiché la sola conoscenza della lingua cinese e/o delle tecniche di interpretazione non sono sufficienti quando entrano in gioco fattori culturali e umani come in questo ambito.

Parole chiave: cinese, cultura cinese, interprete, interprete giuridico, lingua cinese,
\end{abstract}

\section{La mediazione interculturale}

Per mediazione linguistica orale si intende un dialogo tra due interlocutori di lingue diverse, reso possibile dalla presenza di un mediatore/interprete: di fatto un «trialogo» (Mason, 2001).

Perché l'operazione abbia successo, il mediatore/interprete deve saper mettere in campo competenze linguistiche e culturali tali da favorire e promuovere la comunicazione tra i parlanti: com'è ormai generalmente riconosciuto, la sua attività non consiste in una mera trasposizione di parole da un codice linguistico a un altro, 
ma richiede anche competenze comunicative, relazionali, culturali, oltre che doti psicologiche e di comprensione empatica.

"Interpretare [...] può significare anche dover (cercare di) colmare vuoti concettuali e istituzionali” (Rudvin, 2005: 133).

È necessario assicurare che il messaggio arrivi nella forma più vicina all'intenzione con la quale è stato emesso, utilizzando quindi anche canali comunicativi non verbali (i gesti, lo sguardo ecc.).

I codici extralinguistici (cinesica, prossemica, vestemica, oggettemica ecc.) variano da una cultura all'altra (Balboni, 1999: 31). Tutti noi filtriamo l'esperienza quotidiana attraverso le lenti dei cultural frames.

Oltre a interpretare e tradurre, al mediatore viene chiesto di negoziare tra la cultura della società di accoglienza rappresentata dall'istituzione (cultura dominante) e quella minoritaria rappresentata dall'utente straniero (cultura svantaggiata).

L'interprete si fa portavoce, spesso contro la sua stessa volontà, di un'autorità che impone leggi, regole, comportamenti, modelli sociali e culturali alieni (Merlini, 2005).

La sua è dunque una professione di servizio con un forte contenuto sociale, dove considerazioni etiche e deontologiche pongono questioni cruciali quali la neutralità e l'equidistanza rispetto ai suoi interlocutori.

\section{L'interprete di comunità/mediatore interculturale in Italia}

«Interprete», «interprete di comunità», «interprete per i servizi pubblici $\gg$, $\ll$ mediatore linguistico-culturale $»$, «mediatore interculturale $\gg$ : diversi appellativi che corrispondono a una diversa percezione della sua figura. Se infatti l'interprete che lavora in ambito congressuale o commerciale viene visto come un «professionista», il collega che opera nei contesti socio-sanitari o in ambito giuridico viene "abbassato" al rango di «consulente degli immigrati» (Merlini, 2005).

In Italia, il primo riferimento alla figura professionale del mediatore interculturale si trova nella Legge n. 40 del 6/3/1998 che riconosce il diritto al supporto linguistico agli stranieri che entrano in contatto con istituzioni e servizi pubblici quali tribunali, commissariati di polizia, questure, strutture sanitarie, istituzioni scolastiche, enti locali, servizi sociali e così via.

In Italia, l'interpretariato per i servizi pubblici è stato di fatto trascurato fino a tempi molto recenti sia sotto il profilo della formazione che della ricerca, a differenza di quanto avvenuto in altri Paesi, dove da tempo si conoscono i rischi legati a prestazioni di interpretariato non sufficientemente affidabili.

Il mediatore è generalmente uno straniero che vive nel nostro paese e che condivide con il migrante non solo la lingua e la cultura, ma l'esperienza del 
faticoso inserimento nella società italiana riuscendo a orientarsi nei meandri degli iter burocratico-amministrativi e delle convenzioni sociali. Sovente tali mediatori, a parte l'esperienza del vissuto personale, non hanno alcuna formazione specifica.

In particolare, le forze di polizia e i tribunali ricorrono spesso a interpreti non qualificati, per i quali spesso mancano controlli sia sulla qualità delle prestazioni, come pure verifiche sulle credenziali, sulle qualifiche e sui possibili motivi di incompatibilità in base a parentela o affinità religiose, etniche ecc.

Spesso, nell'interpretazione in ambito giudiziario, vi è uno squilibrio di potere a favore di uno dei partecipanti primari, quando ad esempio uno dei due interlocutori è un membro della società del paese ospitante, mentre l'altro è uno straniero appartenente a una minoranza (dialogo asimmetrico). Spesso l'interlocutore appartenente alla cultura dominante ha la facoltà di prendere decisioni che hanno un impatto diretto sulla vita dell'altro interlocutore (Mason, 2001: 148). In situazioni di questo tipo esiste spesso una marcata diversità fra i due anche quanto a mezzi espressivi (Sandrelli, 2005).

L'interprete, trovandosi fisicamente faccia a faccia con le due parti, deve non solo tradurre il messaggio in arrivo, ma anche interagire con esse, coordinare il loro dialogo, aiutarle a superare i problemi dovuti alle sopra citate differenze sociali e culturali. Data la situazione, vi è anche un maggiore coinvolgimento personale, con tutta una serie di implicazioni a livello psicologico (Garwood, 2005).

\section{Mediatore/interprete in ambito giuridico}

Il primo fattore che distingue e rende più difficile l'interpretazione in ambito giudiziario rispetto a quello commerciale o medico, è il setting. In ambito giudiziario è tutto fortemente regolamentato sia per quanto riguarda i comportamenti sia per linguaggio delle persone coinvolte nel processo:

Legal proceedings are among the most strictly regulated activities in all human societies. From arrest to verdict, from charge to indemnification, the road is meticulously stalked out by laws and regulations. At every stop along the way, people with well-defined professional roles play their different parts and carry out their executed duties.[...] For parties to a legal proceeding in one country who come from another country with different norms and conventions, the whole procedure may seem extremely strange and even frightening (Niska, 1995: 294).

L'appropriatezza e l'adeguatezza delle scelte lessicali, operate dall'interprete, è in funzione del riconoscimento non solo dei livelli referenziali ma anche culturali 
[...]. Il lessico, infatti, non è solo una nomenclatura, ma è portatore di nozioni culturali (Straniero, 1999: 129).

In campo giudiziario non si tratta soltanto di trovare delle equivalenze dirette. Il sistema giudiziario che sta dietro i vari termini e concetti giudiziari varia enormemente da una cultura all'altra. Già in Europa vi sono sostanziali differenze tra il diritto romano e il diritto anglosassone, tra un inquisitorial system e un sistema avversativo, ma queste differenze diventano abissali se consideriamo sistemi giudiziari di tipo orientale (Garwood, 2005).

Un altro aspetto di cui tener conto è che l'interprete non deve tradurre soltanto il linguaggio giudiziario utilizzato dalle figure professionali (giudici, avvocati, poliziotti ecc.), ma anche il linguaggio ordinario impiegato dalle altre figure (imputato, testimoni) che talvolta usano versioni non-standard di una lingua.

Factors such as dialects, educational level, register, specialized terms, style, and non verbal cues will influence the interpreter's choice of expression (De Jongh, 1992: 53).

Elementi, questi, che influenzano il modo in cui vediamo una persona e aiutano a determinare il suo livello di credibilità.

Inoltre esistono delle norme etiche a cui attenersi:

un primo dovere è quello di scrupolosa fedeltà. Non dovremo enfatizzare quanto detto dallo straniero, magari aggiungendo qualche parola nostra, o al contrario sminuirne il significato, omettendo qualche frase [...]. L'interprete di tribunale deve invece sempre ricordare che una frase che lui giudica insignificante può successivamente rivelarsi molto importante all'interno del processo. Persino su una parola, a volte, si decide la libertà altrui (Alimenti, 1999: 236-237).

In Paesi in cui esiste un codice deontologico per l'interpretazione giudiziaria viene sempre richiesta la neutralità, l'imparzialità e la riservatezza agli interpreti giudiziari (Gentile et al., 1996). Rudvin mette in dubbio il concetto stesso di neutralità, e vari studi hanno dimostrato che in realtà l'interprete non riesce a restare neutrale (Berk-Seligson, 1990; Jansen, 1995).

Come si può ben immaginare, il lavoro già stressante dell'interprete diventa ancora più stressante per l'interprete giudiziario a causa di tutti i fattori citati: la natura degli argomenti trattati, il pathos umano, le possibili conseguenze di un errore, l'esigenza di neutralità ecc. 


\section{Criticità nell'interpretazione dal cinese}

\subsection{Peculiarità culturali}

In Estremo Oriente, e nella fattispecie in Cina, la cultura può essere definita come high context culture ${ }^{1}$. Il discorso asiatico viene strutturato per progressivi avvicinamenti al punto, con un andamento che potrebbe essere definito a spirale: girare intorno al punto è un modo per metterlo in evidenza con rispetto.

In Cina la società è incentrata sulla partecipazione alle attività del gruppo con un'elevata importanza nel creare e mantenere network interpersonali (关系 guanxi «relazioni»). Anche in ambito comunicativo le relazioni sono considerate gerarchicamente superiori e quindi risulta più importante come si convoglia un messaggio rispetto al suo contenuto essenziale. Differente pure la prossemica: un occidentale tende al contatto fisico diretto con strette di mano e altri gesti di intimità, quali mettere una mano sulla spalla e avvicinare il viso, gesti che creano imbarazzo nell'interlocutore cinese.

Contrapposta è pure l'esternazione delle emozioni: estremamente controllata per i cinesi, più colorita per gli italiani. Il sorriso è universalmente utilizzato per comunicare messaggi positivi, tuttavia nella cultura asiatica è impiegato anche in situazioni nelle quali la persona si sente in imbarazzo: invece di dissentire o rispondere no, spesso i cinesi si limitano a sorridere e a stare in silenzio.

Esiste inoltre tutta una complessa codifica sul tempo e sulla direzione dello sguardo, che varia a seconda del contesto, della gerarchia, della confidenza, del sesso degli interlocutori. Pensiamo a quanto varia nelle culture il tempo concesso allo scambio di sguardi fra due persone di sesso opposto, prima che venga scambiato come proposta erotica ${ }^{2}$. Le mani, insieme al viso, sono probabilmente gli strumenti di comunicazione non verbale più utilizzati e maggiormente codificati, sarebbe dunque difficile fare una panoramica della loro variabilità. Gli italiani enfatizzano le parole con i gesti di mani e braccia. Nella cultura cinese invece gesticolare è considerato indice di poco autocontrollo, maleducazione, mancanza di rispetto.

1 Secondo Hall "una comunicazione, o messaggio, si dice ad alto contesto quando la maggior parte dell'informazione risiede nel contesto o è implicita, mentre assai poco risiede nelle parti esplicite, codificate, trasmesse dal messaggio”, mentre una cultura a basso contesto trasmette la maggior parte delle informazioni attraverso il codice esplicito della lingua (Hall, 1968).

2 L. Maddii, Come possiamo non capirci anche se parliamo la stessa lingua?, IRRE Toscana, Lucia Maddii - IRRE .multicanale poiché si serve di molteplici canali: gestualità, mimica, prossemica, - [PDF Document] (vdocumenti.com) (accesso: 25.11.2019). 
Per quanto riguarda l'intonazione dell'eloquio, i cinesi, abituati a gesti controllati, solitamente impiegano toni di voce anche più alti dei nostri che ci possono apparire fuori luogo ed essere male interpretati. Nella conversazione, spesso è differente la tempistica dei turni di parola. Nelle culture del Mediterraneo è normalmente accettata la sovrapposizione delle voci. I popoli orientali, al contrario, sono infastiditi dalla sovrapposizione e chiedono il rigido rispetto dei turni di parola; nelle conversazioni non sono necessari i riempitivi e il silenzio non mette a disagio gli interlocutori. La postura delle braccia, delle gambe e dei piedi è rilevante: se per gli italiani è segno di poco rispetto levarsi le scarpe, per i cinesi invece è importante sedere in maniera composta.

Differente è pure la concezione del tempo e del rispetto dello stesso. La tradizionale noncuranza con cui gli italiani gestiscono gli appuntamenti può costituire un insulto per l'interlocutore cinese, specie se si tratta di un'autorità pubblica. La lentezza, i rinvii, gli annullamenti, il procrastinare che sono all'ordine del giorno nei nostri processi è impensabile e incomprensibile agli occhi di un cinese.

\subsection{Peculiarità linguistiche}

Il cinese è una macrolingua che può essere considerata una famiglia di lingue (famiglia sinitica) strettamente imparentate. Il cinese parlato si distingue per il suo alto livello di diversità interna. Esistono tra sei e dodici principali gruppi regionali di cinese (a seconda degli schemi di classificazione), tra cui il mandarino è quello principale ( 850 milioni di parlanti). Molti di questi gruppi sono incomprensibili tra loro, mutualmente inintelligibili (Abbiati, 1992).

Il problema della lingua diviene particolarmente importante e complesso, in ambito giudiziario, a partire dalla disponibilità di interpreti per le varianti dialettali.

Sarebbe necessaria una riflessione anche sull'uso del solo interprete di madrelingua (e non professionista) nei procedimenti giudiziari e nelle indagini.

A parte il problema della traducibilità di un termine da una lingua all'altra, esistono anche i problemi legati alle scelte lessicali. Si ha, cioè, lo scontro fra l'esigenza della precisione degli occidentali e la maggiore "tolleranza alla ambiguità" degli orientali.

Rispetto alla lingua italiana (lingua flessiva), la lingua cinese (isolante), si caratterizza per la mancanza di indicatori formali che segnalano il valore grammaticale di un'unità lessicale, come per esempio il numero o il genere, in riferimento ai sostantivi, oppure il tempo e il modo, relativamente alle forme verbali.

Ciò comporta talvolta una serie di difficoltà in serie di analisi dei termini. Ad esempio nei processi, così come nei contratti, viene spesso indicato il termine 
当事人 dangshiren «parte», creando spesso incertezze nell'individuare la specificazione di genere e numero («le parti»). Abbastanza frequente è in cinese l'anisomorfismo semantico, ad esempio 无效 wuxiao corrisponde in italiano a «nullità», «inefficacia», «invalidità».

La polisemia è presente sia nella lingua italiana che nella lingua cinese. Nel lessico specialistico, quale quello giuridico, la polisemia ricorre in misura minore, tuttavia sono presenti alcuni casi, quali 条件 tiaojian «condizione» che oltre a valere come «requisito», «clausola», ha anche l'accezione di 状况 zhuangkuang ovvero «stato generale di una persona». Vanno considerati anche altri aspetti morfosintattici: prendiamo ad esempio l'uso dell'imperativo, molto impiegato in ambito giudiziario. Nella cultura cinese la richiesta, l'ordine, deve essere mitigato fino a essere quasi del tutto velato.

Similarmente, ogni cultura ha codificato regole diverse per negare o dissentire: rispondere «no» a una richiesta o a una domanda di un interlocutore, soprattutto se di una certa autorità, è praticamente vietato nella cultura cinese. Si risponde affermativamente anche se non è vero, perché rispondere «no» sarebbe un'offesa, creando così un circolo vizioso. Esiste anche un diverso utilizzo degli appellativi, dei titoli e delle cariche. Ciò che comunque può causare maggiori incidenti interculturali riguarda il nome e il cognome delle persone. In Cina non si usa chiamare una persona per nome proprio, ma solo per cognome, o per cognome seguito da «Signore» 0 «Signora». Anche all'interno della famiglia non si usa il nome proprio, ma il grado di parentela: gli stessi coniugi si chiamano con degli appellativi o nomignoli. Può capitare che i bambini non conoscano il nome proprio dei genitori e dei nonni. Se pensiamo che in cinese c'è un termine specifico per indicare non solo il grado di parentela, ma anche se è di parte materna o paterna e se è più anziano o più giovane possiamo comprendere come sia possibile questo.

La mancanza di nozioni appropriate sulla cultura e sulla lingua cinese spesso crea, nel dibattito processuale come nelle indagini, una vera e propria babele intorno ai nomi delle persone coinvolte. Esiste anche un diverso uso del registro formale e informale, così come ci sono diversi principi per stabilire chi decide quando si deve passare all'informale e con quali formule.

\section{Il lessico cinese in ambito giudiziario}

L'affermazione del ruolo cruciale della Cina nel mondo globale si è accompagnata a una modernizzazione del diritto e delle istituzioni cinesi, avvenuta in gran parte sotto l'effetto dell'importazione di modelli occidentali. Il lessico giuridico cinese è il risultato di una massiccia opera di traduzione e di studio del diritto occidentale, avvenuta principalmente per il tramite del 
Giappone, e in parte mediante la formazione degli studenti ed esperti cinesi all'estero.

Tra Ottocento e Novecento, tassonomie, costrutti normativi, concetti, termini dei diritti occidentali, veicolati dalla conquista imperialista del Paese, vennero riprodotti in lingua cinese, creando un codice espressivo destinato a sostenere il processo di «modernizzazione» (现代化 xiandaihua) (Timoteo, 2013). Frequente il ricorso a parole derivate mediante l'impiego del suffisso人 ren «persona», quali per esempio 法人 faren «persona giuridica», 本人 benren «rappresentato».

Si stima che in Cina vengano coniate all'incirca mille parole nuove l'anno, la maggior parte delle quali in ambito sociale, economico, e appunto giuridico. $\mathrm{Si}$ tratta di un linguaggio non comune, ricco di tecnicismi, definizioni, chiarimenti e figure retoriche difficilmente comprensibili nella vita di tutti i giorni.

\section{Conclusioni}

A partire dal 2001 con la riforma universitaria che istituisce il corso di laurea triennale di primo livello in Scienze della Mediazione Linguistica numerosi atenei italiani hanno avviato corsi di formazione in questa disciplina dalle denominazioni e curricula più svariati. Tuttavia ad oggi non esistono corsi di laurea con preciso curriculum per interprete giudiziario per le lingue cosiddette minoritarie quali il cinese, l'arabo, il rumeno, il bangla ecc.

Anche per le lingue considerate "principali" quali inglese o francese esistono solamente un paio di corsi ${ }^{3}$. Possiamo ipotizzare che la poca considerazione per l'interprete sociale o comunitario, così come la scarsa retribuzione per questo tipo di prestazione da agiscano da deterrenti nella scelta di questo tipo di professione. Eppure, come evidenziato anche nella recente "Direttiva europea sul diritto alla traduzione e interpretazione nei procedimenti penali", gli interpreti e i traduttori giudiziari devono essere "adeguatamente formati".

Secondo la AssITIG (Associazione Italiana Traduttori e Interpreti Giudiziari), gli interpreti giuridico-giudiziari sono coloro che riversano la lingua di partenza oralmente verso la lingua d'arrivo, e sono specializzati nelle diverse tecniche (simultanea, consecutiva breve con presa d'appunti, chuchotage, trattativa, ivi compresa la traduzione a vista). Per i professionisti italiani

${ }^{3}$ Corso di formazione professionale in Interpretazione Giudiziaria attuato dalla Scuola Superiore per Mediatori Linguistici di Perugia, e Corso interpreti in ambito giuridico-giudiziario: modalità e tecniche di interpretazione di Trieste.

${ }^{4}$ http://data.europa.eu/eli/dir/2010/64/oj, Directive 2010/64/EU of the European Parliament and of the Council of 20 October 2010 on the right to interpretation and translation in criminal proceedings, OJ L 280, 26.10.2010, pp. 1-7. 
è richiesto un certificato attestante livello minimo $\mathrm{C} 1$ della lingua di lavoro straniera secondo il QCER. Per gli stranieri, un certificato attestante livello minimo corrispondente al CELI 4/CILS 3, PLIDA C1 ${ }^{5}$.

Come già ampiamente dimostrato, la conoscenza delle lingue è un requisito necessario, ma non sufficiente, per svolgere questo delicato compito. Inoltre, le competenze (inter)culturali non possono restare limitate al livello «oggettivo» della cultura (saper salutare correttamente, conoscere le regole di comportamento a tavola ecc.) in quanto è proprio la cultura «soggettiva» che crea la maggioranza delle incomprensioni ed è spesso fonte di conflitti, trattandosi spesso di incomprensioni basate su aspetti non consapevoli. Questi aspetti (come i valori, le attitudini, le motivazioni) non si imparano facilmente perché formano i tratti della personalità collettiva di un popolo e, restando per lo più celati sotto la superficie del discorso, sono spesso all'origine di stereotipi e pregiudizi che possono rivelarsi veri e propri ostacoli alla comunicazione (Ulrike A. Kaunzner, 2006).

Come ci si può quindi formare per diventare interpreti professionisti qualificati per l'ambito giudiziario? Dove si impara a affrontare l'emotività dell'accusato, la rabbia, l'uso di espressioni volgari, il rifiuto dell'interprete o dell'avvocato? Dove indirizzare lo sguardo? Come tenere le mani? Si possono incrociare le gambe?

Sono necessari corsi che abbinino nozioni normative e terminologiche del sistema giuridico italiano a tecniche di interpretazione altamente specializzate, a competenze di mediazione interculturale per formare così assistenti linguistici in grado di mediare tra differenti culture, di svolgere lavori di interpretazione consecutiva e simultanea tra l'italiano e una seconda lingua di lavoro 'minoritaria', di tradurre a vista documenti del settore, di assistere l'autorità procedente nel corso di intercettazioni telefoniche, e di saper gestire in modo professionale tutte le situazioni ad alto contenuto emotivo che si presentano.

Auspico pertanto che siano intraprese le iniziative necessarie alla sensibilizzazione degli enti formativi, degli atenei italiani, degli organi governativi e ministeriali competenti, volte alla creazione di percorsi professionalizzanti per la formazione e la qualifica del traduttore giuridico e dell'interprete giudiziario che lavora con le lingue extraeuropee, nonché al riconoscimento dello status giuridico della professione, alla garanzia di condizioni di lavoro ottimali per consentire lo svolgimento dell'operato nell'interesse della giustizia e per tutelare i diritti degli indagati e in generale i principi fondamentali dei diritti umani così come sanciti dagli art. 5 e 6 della Convenzione Europea dei Diritti Umani e Libertà Fondamentali ${ }^{6}$.

5 Pagina informativa della certificazione di italiano CELI dell'Università per Stranieri di Perugia, http://www.cvcl.it/canale.asp?id=33 (accesso: 15.07.2015).

${ }^{6}$ https://www.coe.int/en/web/human-rights-convention/ (accesso: 15.09.2021). 


\section{Bibliografia}

Abbiati, M. (1992): La lingua cinese. Venezia: Cafoscarina.

Alimenti, A. (1999): Il traduttore di tribunale. Libri e Riviste d'Italia, AA.VV., Ministero dei Beni Culturali, Roma, pp. 223-248.

Balboni, P.E. (1999): Parole comuni, culture diverse. Guida alla comunicazione interculturale. Venezia: Marsilio.

Berk-Seligson, S. (1990): The Bilingual Courtroom. Court Interpreters in the Judicial Process. London: The University of Chicago Press.

De Jongh, E. (1992): An Introduction to Court Interpreting: Theory and Practice. Lanham: University Press of America.

Garwood, C. (2005): La formazione dell'interprete di trattativa in ambito giudiziario. In: Russo, M. Mack, G. (a cura di), Interpretazione di trattativa. La mediazione linguistico-culturale nel contesto formativo e professionale, pp. 145-159. Milano: Hoepli.

Gentile, A., Ozolins, U., Vasilakakos, M. (eds) (1996): Liaison Interpreting. A Handbook. Melbourne: Melbourne University Press.

Hall, T.E. (1968): La dimensione nascosta. Mialano: Bompiani.

Jansen, P. (1995): The Role of the Interpreter in Dutch Courtroom Interaction: The Impact of the Situation on Translational Norms. In: Jansen, P. (dir.), Selected Papers of CERA Research Seminars in Translation Studies 1992-1993, pp. 133-155. Leuven : Katholieke Universiteit Leuven.

Maddii L.: Come possiamo non capirci anche se parliamo la stessa lingua?, IRRE Toscana, Lucia Maddii - IRRE .multicanale poiché si serve di molteplici canali: gestualità, mimica, prossemica, - [PDF Document] (vdocumenti.com) (accesso: 25.11.2019).

Mason, I. (ed.) (2001): Triadic Exchanges. Studies in Dialogue Interpreting. Manchester: St. Jerome Publishing.

Merlini, R. (2005): Alla ricerca dell'interprete ritrovato. In: Russo, M., Mack, G. (a cura di), Interpretazione di trattativa. La mediazione linguistico-culturale nel contesto formativo e professionale, pp. 19-40. Milano: Hoepli.

Niska, H. (1995): Just Interpreting: Role Conflicts and Discourse Types. In: Morris, M. (ed.), Translation and the Law, pp. 293-316. Amsterdam-Philadelphia: John Benjamins.

Rudvin, M. (2005): La formazione di interpreti in ambito sociale in Italia e all'estero. In: Russo, M., Mack, G. (a cura di), Interpretazione di trattativa. La mediazione linguistico-culturale nel contesto formativo e professionale, pp. 131-143. Milano: Hoepli.

Sandrelli, A. (2005): La trattativa d'affari: osservazioni generali e strategie didattiche. In: Russo, M., Mack, G. (a cura di), Interpretazione di trattativa. La mediazione linguistico-culturale nel contesto formativo e professionale, pp. 77-91. Milano: Hoepli.

Straniero, S. F. (1999): Verso una sociolinguistica internazionale dell'interpretazione. In: Falbo, C., Russo, M., Straniero, S.F. (a cura di), Interpretazione simultanea e consecutiva: Problemi teorici e metodologie didattiche, pp. 103-139. Milano: Hoepli. 
Timoteo, M. (2013): Il diritto per immagini - aspetti del linguaggio giuridico cinese contemporaneo. In: Pozzo, B. (a cura di), Lingue e Diritto: Oltre l'Europa. Milano: Giuffrè.

\section{Sitografia}

http://www.cvcl.it/canale.asp?id=33 (accesso: 15.07.2015). https://supremepeoplescourtmonitor.com/2018/06/19/how-to-translate-chinesecourt-terminology/ (accesso: 19.09.2018).

https://www.chinalawblog.com/2012/10/chinese-english-translation-of-fiftycommon-legal-words.html (accesso: 19.09.2018).

https://www.coe.int/en/web/human-rights-convention/ (accesso: 15.09.2021). 